\title{
SOME CATEGORICAL ASPECTS OF BCH-ALGEBRAS
}

\author{
MUHAMMAD ANWAR CHAUDHRY and HAFIZ FAKHAR-UD-DIN
}

\author{
Received 14 May 2001
}

\begin{abstract}
We show that the category $\mathrm{BCH}$ of $\mathrm{BCH}$-algebras and $\mathrm{BCH}$-homomorphisms is complete. We also show that it has coequalizers, kernel pairs, and an image factorization system. It is also proved that onto homomorphisms and coequalizers, and monomorphisms and one-to-one homomorphisms coincide, respectively, in $\mathrm{BCH}$. It is shown that MBCI is a coreflexive subcategory of $\mathrm{BCH}$. Regular homomorphisms have been defined and their properties are studied. An open problem has been posed.
\end{abstract}

2000 Mathematics Subject Classification: 06F35, 03G25.

1. Introduction. In 1966, Imai and Iséki introduced two classes of abstract algebras, BCK-algebras and BCI-algebras [10,11]. The notion of a BCI-algebra is a generalization of the notion of a BCK-algebra. These algebras have been studied extensively by various researchers but little attention has been given to their categorical aspects. Some categorical aspects of these algebras have been investigated by Chaudhry and Bhatti [3], Hoo [6], Iséki [12], and Yutani [19, 20, 21].

In 1983, $\mathrm{Hu}$ and $\mathrm{Li}[8,9]$ introduced the notion of a $\mathrm{BCH}$-algebra, which is a generalization of the notions of BCK- and BCI-algebras. They have studied a few properties of these algebras. Certain other properties of these algebras have been studied by Chaudhry [2] and Dudek and Thomys [4]. But, categorical aspects of these algebras have not yet been investigated. The purpose of this paper is to initiate a study about categorical aspects of BCH-algebras.

We will follow standard definitions. Our categorical concepts will be those of standard texts $[1,17]$ to which we refer the reader for the definitions of standard categorical terms. We denote by $\mathrm{BCK}(\mathrm{BCI})$ the category of $\mathrm{BCK}(\mathrm{BCI})$ algebras and $\mathrm{BCK}(\mathrm{BCI})$-homomorphisms. Recall that in both categories, a homomorphism $f: X \rightarrow Y$ means $f\left(x_{1} * x_{2}\right)=f\left(x_{1}\right) * f\left(x_{2}\right)$. This implies $f(0)=$ 0 and $x \leq y$ gives $f(x) \leq f(y)$.

A BCH-algebra is an algebra $(X, *, 0)$ of type $(2,0)$ satisfying the following conditions:

(1) $x \leq x$,

(2) $x \leq y$ and $y \leq x$ imply $x=y$,

(3) $(x * y) * z=(x * z) * y$, where $x \leq y$ if and only if $x * y=0$ (see [8]).

In any $\mathrm{BCH}$-algebra, the following hold:

(4) $x *(x * y) \leq y$ (see [8]), 
(5) $x * 0=0$ implies $x=0$ (see [8]),

(6) $0 *(x * y)=(0 * x) *(0 * y)$ (see [4]),

(7) $x * 0=x$ (see [4]),

(8) $(x * y) * x=0 * y$ (see [8]),

(9) $x \leq y$ implies $0 * x=0 * y$ (see [2]).

It is known that every BCI-algebra is a $\mathrm{BCH}$-algebra but not conversely $[8,9]$. A BCH-algebra $X$ is called proper if it is not a BCI-algebra. It is known that proper BCH-algebras exist. It is known [9] that in a BCH-algebra, $x \leq y$ implies $x * z \leq y * z$ and $x \leq y$ implies $z * y \leq z * x$ do not hold. The set $X_{+}\{x: x \in$ $X, 0 \leq x\}$ is called the BCA-part of a BCH-algebra $X$. It is known that $X_{+}$is an ideal of $X$ (see [2]).

A BCH-homomorphism $f: X \rightarrow Y$ means $f\left(x_{1} * x_{2}\right)=f\left(x_{1}\right) * f\left(x_{2}\right)$. This implies $f(0)=0$ and $x \leq y$ gives $f(x) \leq f(y)$. The category of BCH-algebras and $\mathrm{BCH}$-homomorphisms is denoted by $\mathrm{BCH}$. We will denote a general category by $K$, its objects by $|K|$, and the set of morphisms from a $K$-object $A$ into a $K$-object $B$ by $K(A, B)$.

Our notions of BCK-algebras will be as developed in [14, 15], those of BCIalgebras will be as in $[5,7,13,16,18]$, and those of $\mathrm{BCH}$-algebras will be as in $[2,4,8,9]$.

2. Limits in $\mathrm{BCH}$. In this section, we show that the category $\mathrm{BCH}$ has arbitrary products and equalizers.

THEOREM 2.1. The category BCH has arbitrary products.

Proof. Let $\left\{X_{\alpha}: \alpha \in J\right\}$ be a family of BCH-algebras. Let $\prod_{\alpha \in J} X_{\alpha}=\{f \mid f$ : $J \rightarrow \bigcup_{\alpha \in J} X_{\alpha}$ is a function, $f(\alpha) \in X_{\alpha}$ for all $\left.\alpha \in J\right\}$.

We define the binary operation $*$ in $\prod_{\alpha \in I} X_{\alpha}$ by $(f * g)(\alpha)=f(\alpha) * g(\alpha)$ for all $\alpha \in J$. Then routine calculations give that $\prod_{\alpha \in J} X_{\alpha}$ is a $\mathrm{BCH}$-algebra under this operation with zero as zero function given by $0(\alpha)=0_{\alpha}, 0_{\alpha}$ being the zero of $X_{\alpha}$.

Further, the mapping $\operatorname{pr}_{\alpha}: \prod_{\alpha \in J} X_{\alpha} \rightarrow X_{\alpha}, \alpha \in J$, defined by $\operatorname{pr}_{\alpha}(f)=f(\alpha)$, for all $\alpha \in J$, is a BCH-homomorphism. Further, for all $X \in|\mathrm{BCH}|, f_{\alpha} \in \mathrm{BCH}(X$, $\left.X_{\alpha}\right), \alpha \in J$, the mapping $\psi: X \rightarrow \prod_{\alpha \in J} X_{\alpha}$ defined by $(\psi(x))(\alpha)=f_{\alpha}(x)$ for all $x \in X, \alpha \in J$ is the unique BCH-homomorphism making the following diagram commutative:

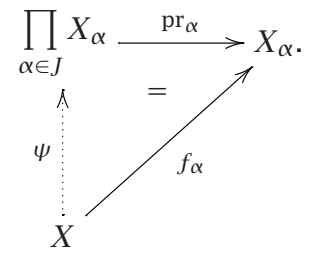

This completes the proof. 
THEOREM 2.2. The category BCH has equalizers.

Proof. Let $f, g \in \operatorname{BCH}(X, Y)$. Define $Z=\{x: x \in X, f(x)=g(x)\}$. It is easy to verify that $Z$ is a subalgebra of $X$. Let $i: Z \rightarrow X$ be imbedding of $Z$ into $X$, given by $i(z)=z$ for all $z \in Z$. Obviously, $i \in \mathrm{BCH}(Z, X)$ and satisfies $f \circ i=$ $g \circ i$. Further, let $C \in|\mathrm{BCH}|$ and let $h \in \operatorname{BCH}(C, X)$ be such that $f \circ h=g \circ h$. Then the function $\psi: C \rightarrow Z$ given by $\psi(c)=h(c)$ is well defined because $f(h(c))=g(h(c))$ implies $h(c) \in Z$ for all $c \in C$. Further, $\psi \in \operatorname{BCH}(C, Z)$ and is the unique such $\mathrm{BCH}$-homomorphism making the following diagram commutative:

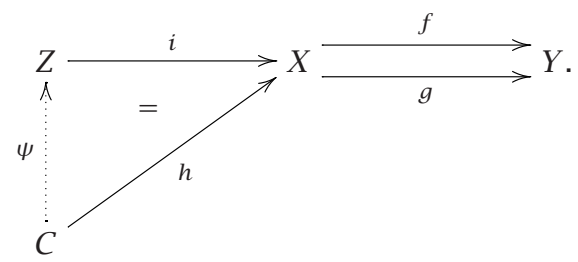

Thus, $i=\operatorname{eq}(f, g)$. This completes the proof.

Since a category $K$ with arbitrary products and equalizers has arbitrary limits [17], we have the following theorem.

THEOREM 2.3. The category BCH has arbitrary limits and is complete.

We now pose the following problem.

OPEN PROBLEM. Whether the category BCH has coproducts or not.

3. Monomorphisms, epimorphisms, and coequalizers in $\mathrm{BCH}$. In this section, we show that $\mathrm{BCH}$ has kernel pairs and coequalizers. It is also proved that onto homomorphisms and coequalizers, and monomorphisms and oneto-one homomorphisms coincide, respectively, in BCH. Further, onto homomorphisms are epimorphisms but the converse is not obvious. However, we give a partial solution of the converse.

We now prove the following propositions which will be used in the sequel. Their proofs are carried out on the lines of the proofs given by Hoo [6] for similar results about the categories BCK and BCI.

Proposition 3.1. In BCH, onto homomorphisms are epimorphisms.

Proof. Let $f \in \mathrm{BCH}(X, Y)$ be an onto homomorphism. Let $Z \in|\mathrm{BCH}|$ and let $g_{1}, g_{2} \in \mathrm{BCH}(Y, Z)$ be such that $g_{1} \circ f=g_{2} \circ f$. We will prove that $g_{1}=g_{2}$. Now, $g_{1} \circ f=g_{2} \circ f$ implies $g_{1}(f(x))=g_{2}(f(x))$ for all $x \in X$. Let $y \in Y$. Since $f$ is onto, so there exists an $x_{1} \in X$ such that $f\left(x_{1}\right)=y$. Hence, $g_{1}\left(f\left(x_{1}\right)\right)=$ $g_{2}\left(f\left(x_{1}\right)\right)$ or $g_{1}(y)=g_{2}(y)$ for all $y \in Y$, that is, $g_{1}=g_{2}$. This completes the proof. 
Proposition 3.2. In BCH, one-to-one homomorphisms are monomorphisms.

Proof. Let $f \in \mathrm{BCH}(X, Y)$ be a one-to-one homomorphism. Let $Z \in|\mathrm{BCH}|$ and let $g_{1}, g_{2} \in \mathrm{BCH}(Z, X)$ be such that $f \circ g_{1}=f \circ g_{2}$ which gives $f\left(g_{1}(z)\right)=$ $f\left(g_{2}(z)\right)$ for all $z \in Z$. Since $f$ is a one-to-one homomorphism, it follows that $g_{1}(z)=g_{2}(z)$ for all $z \in Z$, that is, $g_{1}=g_{2}$. This completes the proof.

We now prove the following results.

THEOREM 3.3. The category BCH has kernel pairs.

Proof. Let $f \in \operatorname{BCH}(X, Y)$. Let $Z=\left\{\left(x_{1}, x_{2}\right): x_{1}, x_{2} \in X, f\left(x_{1}\right)=f\left(x_{2}\right)\right\}$. Obviously, $Z$ is a subalgebra of the product algebra $X \times X$. Let $p_{1}, p_{2}: Z \rightarrow X$ be defined by $p_{1}\left(x_{1}, x_{2}\right)=x_{1}$ and $p_{2}\left(x_{1}, x_{2}\right)=x_{2}$ for all $\left(x_{1}, x_{2}\right) \in Z$. It is easy to verify that $p_{1}$ and $p_{2}$ are BCH-homomorphisms and satisfy $f \circ p_{1}=f \circ p_{2}$.

We claim that the pair $\left(p_{1}, p_{2}\right)$ is a kernel pair of $f$. Let $g_{1}, g_{2} \in \operatorname{BCH}(D, X)$ be such that $f \circ g_{1}=f \circ g_{2}$. We take $g: D \rightarrow Z$ as

$$
g(d)=\left(g_{1}(d), g_{2}(d)\right) \quad \forall d \in D
$$

Now, $g$ is well defined because $f \circ g_{1}=f \circ g_{2}$ gives $f\left(g_{1}(d)\right)=f\left(g_{2}(d)\right)$, which gives $\left(g_{1}(d), g_{2}(d)\right) \in Z$. Further

$$
\begin{aligned}
g\left(d_{1} * d_{2}\right) & =\left(g_{1}\left(d_{1} * d_{2}\right), g_{2}\left(d_{1} * d_{2}\right)\right) \\
& =\left(g_{1}\left(d_{1}\right) * g_{1}\left(d_{2}\right), g_{2}\left(d_{1}\right) * g_{2}\left(d_{2}\right)\right) \\
& =\left(g_{1}\left(d_{1}\right), g_{2}\left(d_{1}\right)\right) *\left(g_{1}\left(d_{2}\right), g_{2}\left(d_{2}\right)\right) \\
& =g\left(d_{1}\right) * g\left(d_{2}\right) .
\end{aligned}
$$

Thus, $g \in \mathrm{BCH}(D, Z)$ and satisfies $p_{1} \circ g=g_{1}$ and $p_{2} \circ g=g_{2}$.

Now, we prove that $g$ is unique. Let $g^{\prime} \in \mathrm{BCH}(D, Z)$ be such that $p_{1} \circ g^{\prime}=g_{1}$ and $p_{2} \circ g^{\prime}=g_{2}$. Let $g^{\prime}(d)=\left(a^{d}, b^{d}\right)$.

Now, $p_{1} \circ g^{\prime}=p_{1} \circ g$ gives $p_{1}\left(a^{d}, b^{d}\right)=p_{1}\left(g_{1}(d), g_{2}(d)\right)$ which gives $a^{d}=$ $g_{1}(d)$. Similarly, we can prove that $b^{d}=g_{2}(d)$. Thus, $g^{\prime}(d)=\left(g_{1}(d), g_{2}(d)\right)=$ $g(d)$ for all $d \in D$. Hence, $g$ is unique. This completes the proof.

THEOREM 3.4. Let $f \in \mathrm{BCH}(X, Y)$ be onto, then $f$ is a coequalizer.

Proof. Let $f: X \rightarrow Y$ be onto and let $X \times X$ be the product BCH-algebra of $X$ with itself. Let $Z=\left\{\left(x_{1}, x_{2}\right): x_{1}, x_{2} \in X, f\left(x_{1}\right)=f\left(x_{2}\right)\right\}$. Obviously, $Z$ is a subalgebra of $X \times X$ and thus $Z \in|\mathrm{BCH}|$. Let $p_{1}, p_{2}: Z \rightarrow X$ be defined by

$$
p_{1}\left(x_{1}, x_{2}\right)=x_{1}, \quad p_{2}\left(x_{1}, x_{2}\right)=x_{2} \quad \forall\left(x_{1}, x_{2}\right) \in Z
$$

It is easy to verify that $p_{1}$ and $p_{2}$ are BCH-homomorphisms and satisfy $f \circ p_{1}=$ $f \circ p_{2}$. Let $g \in \operatorname{BCH}(X, C)$ be such that $g \circ p_{1}=g \circ p_{2}$. Since $f: X \rightarrow Y$ is onto, so for any $y \in Y$, there exists an $x \in X$ such that $f(x)=y$. We define $h: Y \rightarrow C$ by $h(y)=g(x)$, where $f(x)=y$ for all $y \in Y$. To show that $h$ is well defined, we 
consider $f\left(x_{1}\right)=f\left(x_{2}\right)=y$, say. This implies $\left(x_{1}, x_{2}\right) \in Z$ and $g \circ p_{1}=g \circ p_{2}$ gives $g\left(x_{1}\right)=g\left(x_{2}\right)$. Thus, $h(y)=g\left(x_{1}\right)=g\left(x_{2}\right)$. Further, let $y_{1}, y_{2} \in Y$, then there exist $x_{1}, x_{2} \in X$ such that $f\left(x_{1}\right)=y_{1}$ and $f\left(x_{2}\right)=y_{2}$, and hence $g\left(x_{1}\right)=h\left(y_{1}\right)$ and $g\left(x_{2}\right)=h\left(y_{2}\right)$. Further, $y_{1} * y_{2}=f\left(x_{1}\right) * f\left(x_{2}\right)=f\left(x_{1} *\right.$ $\left.x_{2}\right)$. Hence, $h\left(y_{1} * y_{2}\right)=g\left(x_{1} * x_{2}\right)=g\left(x_{1}\right) * g\left(x_{2}\right)=h\left(y_{1}\right) * h\left(y_{2}\right)$. Thus, $h \in \operatorname{BCH}(Y, C)$ and obviously it satisfies $h \circ f=g$. The uniqueness of $h$ follows from the fact that $f$ is an onto homomorphism and Proposition 3.1. Thus, $f=\operatorname{coeq}\left(p_{1}, p_{2}\right)$.

DEFINITION 3.5. Let $f: X \rightarrow Y$ be a homomorphism of BCH-algebras. The kernel of $f$ is defined by $\{x \in X: f(x)=0\}$ and is denoted by Ker $f$.

Obviously, $0 \in \operatorname{Ker} f$ and thus $\operatorname{Ker} f$ is nonempty.

DEFINITION 3.6. A nonempty subset $A$ of a BCH-algebra $X$ is called an ideal of $X$ if (i) $0 \in A$ and (ii) $y * x \in A, x \in A$ imply that $y \in A$.

A nonempty subset $A$ of a BCH-algebra $X$ is called a closed ideal of $X$ if (i) $0 * x \in A$ for all $x \in A$ and (ii) $y * x \in A, x \in A$ imply that $y \in A$.

It is known that every ideal is not necessarily closed. In the sequel, by an ideal, we will always mean a closed ideal.

DEFINITION 3.7. A BCH-algebra $X$ is called medial if $x *(x * y)=y$ for all $x, y \in X$.

It is known that a medial BCH-algebra is a medial BCI-algebra [4]. MBCI denotes the category of medial BCI-algebras and BCI-homomorphisms.

Proposition 3.8. Let $f \in \mathrm{BCH}(X, Y)$. Then $\operatorname{Ker} f$ is an ideal of $X$.

Proof. Let $x \in \operatorname{Ker} f$. Then $f(0 * x)=f(0) * f(x)=0 * 0=0$. Thus, $0 * x \in$ Ker $f$ for all $x \in \operatorname{Ker} f$. Next, suppose $x * y, y \in \operatorname{Ker} f$. Then

$$
0=f(x * y)=f(x) * f(y)=f(x) * 0=f(x) .
$$

Hence, $x \in \operatorname{Ker} f$. This proves that $\operatorname{Ker} f$ is an ideal of $X$.

THEOREM 3.9. Let $X, Y, Z \in|\mathrm{BCH}|$ and let $f \in \mathrm{BCH}(X, Y)$ be onto. Let $g \in$ $\operatorname{BCH}(X, Z)$ be such that $\operatorname{Ker} f \subseteq \operatorname{Ker} g$. Then there exists a unique $h \in \mathrm{BCH}(Y, Z)$ such that $h \circ f=g$.

Proof. Let $D=\left\{\left(x_{1}, x_{2}\right): x_{1}, x_{2} \in X, f\left(x_{1}\right)=f\left(x_{2}\right)\right\}$. Then $D$ is a subalgebra of $X \times X$. Define $p_{1}, p_{2}: D \rightarrow X$ as $p_{1}\left(x_{1}, x_{2}\right)=x_{1}$ and $p_{2}\left(x_{1}, x_{2}\right)=x_{2}$. Obviously, $p_{1}$ and $p_{2}$ are BCH-homomorphisms and satisfy $f \circ p_{1}=f \circ p_{2}$. Let $\left(x_{1}, x_{2}\right) \in D$. Then $f\left(x_{1}\right)=f\left(x_{2}\right)$ which gives that $x_{1} * x_{2} \in \operatorname{Ker} f$ and $x_{2} * x_{1} \in \operatorname{Ker} f$. Since Ker $f \subseteq \operatorname{Ker} g$, it follows that $g\left(x_{1}\right)=g\left(x_{2}\right)$. Thus, $g \circ p_{1}=g \circ p_{2}$. Since every coequalizer in a category $K$ is a coequalizer of its kernel pairs and the $\mathrm{BCH}$-algebra $D$, together with the projections $p_{1}$ and $p_{2}$, is a kernel pair of $f$, then $f=\operatorname{coeq}\left(p_{1}, p_{2}\right)$. So by the definition of a coequalizer, there exists a unique homomorphism $h: Y \rightarrow Z$ making the following diagram 
commutative, that is, $h \circ f=g$ :

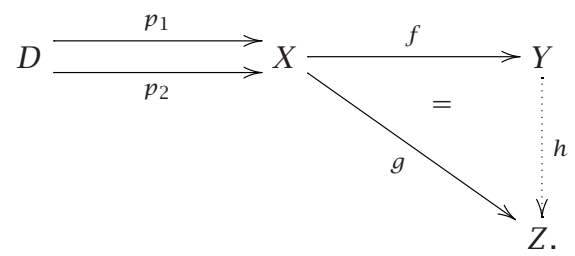

This completes the proof.

In the coming results, we will need the definitions of regular congruences and quotient algebras and for these, we refer the reader to [2].

THEOREM 3.10. Every coequalizer in BCH is an onto homomorphism.

Proof. Let $f \in \mathrm{BCH}(X, Y)$ be a coequalizer. Since every coequalizer in a category $K$ is a coequalizer of its kernel pairs and the BCH-algebra $Z=$ $\left\{\left(x_{1}, x_{2}\right): x_{1}, x_{2} \in X, f\left(x_{1}\right)=f\left(x_{2}\right)\right\}$, together with the projections defined in Theorem 3.4, is a kernel pair of $f$, therefore $f=\operatorname{coeq}\left(p_{1}, p_{2}\right)$. We further note that $Z$ is a regular congruence on $X$ generated by $\operatorname{Ker} f=K$ because

$$
\begin{aligned}
Z & =\left\{\left(x_{1}, x_{2}\right): x_{1}, x_{2} \in X, f\left(x_{1}\right)=f\left(x_{2}\right)\right\} \\
& =\left\{\left(x_{1}, x_{2}\right): f\left(x_{1} * x_{2}\right)=0=f\left(x_{2} * x_{1}\right)\right\} \\
& =\left\{\left(x_{1}, x_{2}\right): x_{1} * x_{2} \in K, x_{2} * x_{1} \in K\right\} .
\end{aligned}
$$

Let $X / K$ be the corresponding quotient BCH-algebra and let nat: $X \rightarrow X / K$ be defined by nat $(x)=[x]_{K}$, where $[x]_{K}=\{y \in X:(x, y) \in Z\}$. We consider $\operatorname{nat}\left(x_{1} * x_{2}\right)=\left[x_{1} * x_{2}\right]_{K}=\left[x_{1}\right]_{K} *\left[x_{2}\right]_{K}=\operatorname{nat}\left(x_{1}\right) *$ nat $\left(x_{2}\right)$. Thus, nat $\in$ $\operatorname{BCH}(X, X / K)$. Further, nato $p_{1}\left(x_{1}, x_{2}\right)=\operatorname{nat}\left(x_{1}\right)=\left[x_{1}\right]_{K}=\left[x_{2}\right]_{K}=\operatorname{nat}\left(x_{2}\right)=$ nat $\circ p_{2}\left(x_{1}, x_{2}\right)$ for all $\left(x_{1}, x_{2}\right) \in Z$. Thus, nat $\circ p_{1}=$ nat $\circ p_{2}$. Since $f=\operatorname{coeq}\left(p_{1}\right.$, $\left.p_{2}\right)$, there exists a unique $\psi \in \operatorname{BCH}(Y, X / K)$ such that $\psi \circ f=$ nat, that is, the following diagram commutes:

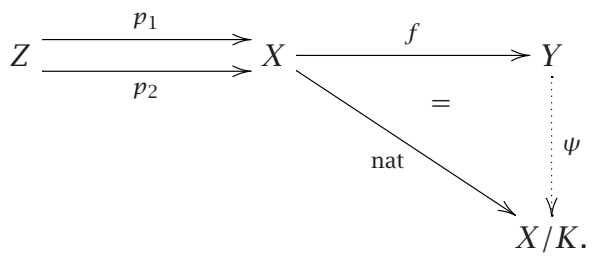

Let $x \in \operatorname{Ker}\left(\right.$ nat). Then nat $(x)=[0]_{K}$. But, nat $(x)=[x]_{K}$. Thus, $(0, x) \in Z$, which gives $x * 0=x \in \operatorname{Ker} f$. Obviously, nat is onto. Thus, by Theorem 3.9, 
there exists a unique $g \in \mathrm{BCH}(X / K, Y)$ such that $g \circ$ nat $=f$, that is, the following diagram commutes:

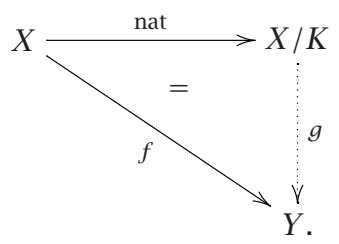

Now

$$
\begin{gathered}
\psi \circ g \circ \text { nat }=\psi \circ f=\text { nat }=I_{X / K} \circ \text { nat, } \\
g \circ \psi \circ f=g \circ \text { nat }=f=I_{Y} \circ f .
\end{gathered}
$$

Since nat is onto and $f$ is a coequalizer, both are epimorphisms. Hence, (3.9) gives that

$$
\psi \circ g=I_{X / K}, \quad g \circ \psi=I_{Y} .
$$

Thus, $\psi \in \mathrm{BCH}(Y, X / K)$ is an isomorphism. Since nat is onto, $f$ is onto.

Combining Theorems 3.4 and 3.10, we get that coequalizers and onto homomorphisms coincide in the category $\mathrm{BCH}$.

THEOREM 3.11. Let $f \in \mathrm{BCH}(X, Y)$. Then $f$ is a monomorphism if and only if $\operatorname{Ker} f=\{0\}$.

Proof. Let $f \in \mathrm{BCH}(X, Y)$. Obviously, $\operatorname{Ker} f \in|\mathrm{BCH}|$. Define $g, h: \operatorname{Ker} f \rightarrow$ $X$ as $g(x)=x$ and $h(x)=0$. Then $g, h \in \operatorname{BCH}(\operatorname{Ker} f, X)$ and satisfy $f \circ g=f \circ h$. Since $f$ is a monomorphism, it follows that $g=h$, that is, $g(x)=h(x)$ for all $x \in \operatorname{Ker} f$, which gives $x=0$. Thus, $\operatorname{Ker} f=\{0\}$.

Conversely, suppose that Ker $f=\{0\}$. Let $x_{1}, x_{2} \in X$ be such that $f\left(x_{1}\right)=$ $f\left(x_{2}\right)$, which gives $f\left(x_{1} * x_{2}\right)=0=f\left(x_{2} * x_{1}\right)$. Thus, $x_{1} * x_{2} \in \operatorname{Ker} f$ and $x_{2} * x_{1} \in \operatorname{Ker} f$. Since $\operatorname{Ker} f=\{0\}$, it follows that $x_{1}=x_{2}$. Thus, $f$ is one-toone. Now, Proposition 3.2 gives that $f$ is a monomorphism. Hence the theorem follows.

THEOREM 3.12. In BCH, monomorphisms are one-to-one homomorphisms.

Proof. Let $f \in \operatorname{BCH}(X, Y)$ be a monomorphism. Let $a_{1}, a_{2} \in X$ be such that $f\left(a_{1}\right)=f\left(a_{2}\right)$. Let $Z=\left\{\left(x_{1}, x_{2}\right): x_{1}, x_{2} \in X, f\left(x_{1}\right)=f\left(x_{2}\right)\right\}$. Then $Z$ is a subalgebra of the product algebra $X \times X$. Let $p_{1}, p_{2}: Z \rightarrow X$ be defined by (3.3).

It is easy to verify that $p_{1}$ and $p_{2}$ are BCH-homomorphisms satisfying $f \circ$ $p_{1}=f \circ p_{2}$. 
Since $f$ is a monomorphism, it follows that $p_{1}=p_{2}$. Now, $f\left(a_{1}\right)=f\left(a_{2}\right)$ gives that $\left(a_{1}, a_{2}\right) \in Z$. Thus, $p_{1}\left(a_{1}, a_{2}\right)=p_{2}\left(a_{1}, a_{2}\right)$, which gives $a_{1}=a_{2}$. This proves that $f$ is a one-to-one homormorphism.

REMARK 3.13. Combining Proposition 3.2 and Theorem 3.12, we get that, in the category $\mathrm{BCH}$, monomorphisms and one-to-one homomorphisms coincide.

THEOREM 3.14. Let $f \in \operatorname{BCH}(X, Y)$ be an epimorphism. Then $f$ is an onto homomorphism whenever $Y$ is a medial BCH-algebra.

Proof. Let $f \in \mathrm{BCH}(X, Y)$ be an epimorphism and $Y$ a medial BCH-algebra. Then it is easy to verify that $f(X)=\{f(x): x \in X\}$ is a subalgebra, and hence an ideal of $Y$, because every subalgebra of a medial BCH-algebra $Y$ is an ideal of $Y$, see [7]. So it follows that $Y / f(X)$ is well defined. Define $g, h: Y \rightarrow Y / f(X)$ as $g(y)=[y]_{f(X)}$ and $h(y)=[0]_{f(X)}$. Easy calculations give that $g$ and $h$ are BCH-homomorphisms and satisfy $g \circ f=h \circ f$. Since $f$ is an epimorphism, it follows that $g=h$. Thus, $g(y)=h(y)$ for all $y \in Y$, which gives $[y]_{f(X)}=$ $[0]_{f(X)}$. Thus, $y * 0=y \in f(X)$. Hence, $Y \subseteq f(X)$, proving that $f$ is onto.

COROLLARY 3.15. Let $X$ be a BCH-algebra and let $f \in \mathrm{BCH}\left(X, X / X_{+}\right)$be an epimorphism. Then $f$ is onto.

Proof. It follows from Theorem 3.14 because $X / X_{+}$is always medial [2].

THEOREM 3.16. Let $X, Y, Z \in|\mathrm{BCH}|, g \in \mathrm{BCH}(X, Z)$, and let $h \in \mathrm{BCH}(Y, Z)$ be a monomorphism with $\operatorname{Im}(g) \subseteq \operatorname{Im}(h)$. Then there exists a unique $f \in$ $\mathrm{BCH}(X, Y)$ such that $g=h \circ f$.

Proof. Let $x \in X$. Then $g(x) \in \operatorname{Im}(g) \subseteq \operatorname{Im}(h)$. Since $h$ is a monomorphism, so it is one-to-one. Thus, there exists a unique $y \in Y$ such that $h(y)=$ $g(x)$. Now, we define $f: X \rightarrow Y$ as $f(x)=y$ if $h(y)=g(x)$. First, we prove that $f$ is a homomorphism. Let $x_{1}, x_{2} \in X$. Then there exist $y_{1}, y_{2} \in Y$ such that $h\left(y_{1}\right)=g\left(x_{1}\right)$ and $h\left(y_{2}\right)=g\left(x_{2}\right)$. Further, $h\left(y_{1} * y_{2}\right)=h\left(y_{1}\right) * h\left(y_{2}\right)=$ $g\left(x_{1}\right) * g\left(x_{2}\right)=g\left(x_{1} * x_{2}\right)$. Thus, $f\left(x_{1} * x_{2}\right)=y_{1} * y_{2}=f\left(x_{1}\right) * f\left(x_{2}\right)$. Let $x \in X$. Then there exists a unique $y \in Y$ such that $h(y)=g(x)$ which implies $f(x)=y$. Now, $h \circ f(x)=h(f(x))=h(y)=g(x)$ for all $x \in X$. Thus, $h \circ f=g$. The uniqueness of $f$ follows from the fact that $h$ is a monomorphism. This completes the proof.

THEOREM 3.17. Let $f \in \mathrm{BCH}(X, Y)$ and let $i: f(X) \rightarrow Y$ be an imbedding of $f(X)$ into $Y$, where $f(X)=\{f(x): x \in X\}$. Then there exists exactly one epimorphism $\bar{f} \in \mathrm{BCH}(X, f(X))$ such that $i \circ \bar{f}=f$. If $f$ is a monomorphism, then $\bar{f}$ is a monomorphism.

Proof. Define $\bar{f}: X \rightarrow f(X)$ as $\bar{f}(x)=f(x)$ for all $x \in X$. Obviously, $\bar{f} \in \mathrm{BCH}(X, f(X))$ and satisfies $i \circ \bar{f}=f$. The uniqueness of $\bar{f}$ follows from the fact that $i$ is a monomorphism. Let $f$ be a monomorphism. We prove that 
$\bar{f}$ is a monomorphism. Let $C \in|\mathrm{BCH}|$ and let $h_{1}, h_{2} \in \mathrm{BCH}(C, X)$ be such that $\bar{f} \circ h_{1}=\bar{f} \circ h_{2}$. Then $i \circ \bar{f} \circ h_{1}=i \circ \bar{f} \circ h_{2}$, which gives $f \circ h_{1}=f \circ h_{2}$. Hence, $h_{1}=h_{2}$ because $f$ is a monomorphism. This proves that $\bar{f}$ is a monomorphism.

THEOREM 3.18. The category BCH has coequalizers.

Proof. Let $f, g \in \mathrm{BCH}(X, Y)$. Let $R$ be the minimum regular congruence containing $\bar{R}=\{(f(x), g(x)): x \in X\}$. Such a regular congruence exists and is the intersection of all regular congruences on $Y$ containing $\bar{R}$. The quotient algebra $Y / R$ is a $\mathrm{BCH}$-algebra and the mapping nat : $Y \rightarrow Y / R$ defined as nat $(y)=[y]_{R}$ is an onto BCH-homomorphism.

We show that nat $=\operatorname{coeq}(f, g)$. Obviously, nat॰ $f(x)=\operatorname{nat}(f(x))=$ $[f(x)]_{R}=[g(x)]_{R}=$ nat $\circ g(x)$ for all $x \in X$ because $\bar{R} \subseteq R$ and $(f(x), g(x)) \in$ $\bar{R}$ for all $x \in X$. Thus, nat $\circ f=$ nat $\circ$. Let $h \in \mathrm{BCH}(Y, Z)$ be such that $h \circ g=$ $h \circ f$. We now define $\psi: Y / R \rightarrow Z$ by $\psi\left([y]_{R}\right)=h(y)$. To show that $\psi$ is well defined, we suppose that $\left[y_{1}\right]_{R}=\left[y_{2}\right]_{R}$. Thus, $\left(y_{1}, y_{2}\right) \in R$. Further, $R_{1}=\left\{\left(y, y^{\prime}\right): y, y^{\prime} \in Y, h(y)=h\left(y^{\prime}\right)\right\}$ is the regular congruence on $Y$ generated by Ker $h$. We note that $h(f(x))=h(g(x))$ for all $x \in X$. Thus, $(f(x), g(x)) \in R_{1}$. Hence, $R \subseteq R_{1}$. Now, $\left(y_{1}, y_{2}\right) \in R$ gives $\left(y_{1}, y_{2}\right) \in R_{1}$. Thus, $h\left(y_{1}\right)=h\left(y_{2}\right)$, which gives that $\psi$ is well defined. Further, $\psi\left(\left[y_{1}\right]_{R} *\left[y_{2}\right]_{R}\right)=$ $\psi\left(\left[y_{1} * y_{2}\right]_{R}\right)=h\left(y_{1} * y_{2}\right)=h\left(y_{1}\right) * h\left(y_{2}\right)=\psi\left(\left[y_{1}\right]_{R}\right) * \psi\left(\left[y_{2}\right]_{R}\right)$. Thus, $\psi \in \mathrm{BCH}(Y / R, Z)$ and obviously it satisfies $\psi \circ$ nat $=h$, that is, it makes the following diagram commutative:

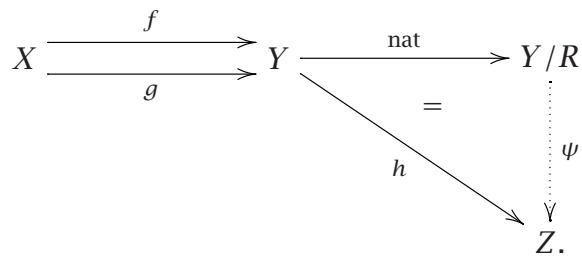

The uniqueness of $\psi$ follows from the fact that nat is onto and hence an epimorphism in $\mathrm{BCH}$. This completes the proof.

4. Image factorization system and certain other notions. In this section, we show that $\mathrm{BCH}$ has an image factorization system and that $\mathrm{MBCI}$, the category of medial BCI-algebras and BCI-homomorphisms, is a coreflexive subcategory of $\mathrm{BCH}$. Further, we will define regular homomorphisms and discuss some of their properties. For the definition of an image factorization system, we refer the reader to [1]. Further, we state the following result which will be used in the sequel.

Proposition 4.1 (see [1, page 40]). Let $K$ be a category in which every morphism factors as a coequalizer followed by a monomorphism. Then the system (coequalizers, monomorphisms) yields an image factorization system in $\mathrm{K}$. 
We now use this result to prove the following theorem.

THEOREM 4.2. The system (coequalizers, monomorphism) form an image factorization system in $\mathrm{BCH}$.

Proof. Let $f \in \operatorname{BCH}(X, Y)$. Then it is easy to verify that $f(X)=\{f(x)$ : $x \in X\}$ is a BCH-algebra. Thus, $f(X) \in|\mathrm{BCH}|$. Now, we define $e: X \rightarrow f(X)$ as $e(x)=f(x)$. Obviously, $e$ is an onto homomorphism. Then by Theorem 3.4, $e$ is a coequalizer. Further, we define $m: f(X) \rightarrow Y$ as $m(y)=y$ for all $y \in f(X)$. Obviously, $m$ is a one-to-one homomorphism. Thus, by Proposition 3.2, $m$ is a monomorphism. Also note that $m \circ e(x)=m(e(x))=e(x)=f(x)$ for all $x \in X$, that is, $m \circ e=f$. This completes the proof.

Obviously, $\mathrm{MBCI}$ is a subcategory of $\mathrm{BCH}$. We now prove the following result.

THEOREM 4.3. The category MBCI is a coreflexive subcategory of BCH.

Proof. Let $X \in|\mathrm{BCH}|$. Then $X / X_{+}$is a MBCI-algebra (see [2]). Thus, $X / X_{+} \in$ $\mid$ MBCI $\mid$. We define nat $_{X}: X \rightarrow X / X_{+}$by nat ${ }_{X}(x)=[x]_{X_{+}}$. Obviously, nat ${ }_{X} \in$ $\operatorname{BCH}\left(X, X / X_{+}\right)$. Let $Y \in|\mathrm{MBCI}|$ and $f \in \mathrm{BCH}(X, Y)$. Let $x \in X_{+}$, then $0 * x=0$ gives $0=f(0)=f(0 * x)=f(0) * f(x)=0 * f(x)$. Therefore, $x \in X_{+}$implies $f(x) \in Y_{+}$.

We now define $g: X / X_{+} \rightarrow Y$ by $g\left([x]_{X_{+}}\right)=f(x)$. First of all, we show that $g$ is well defined. Let $\left[x_{1}\right]_{X_{+}}=\left[x_{2}\right]_{X_{+}}$. Thus, $x_{1} * x_{2} \in X_{+}$and $x_{2} * x_{1} \in X_{+}$, which gives $f\left(x_{1} * x_{2}\right) \in Y_{+}$and $f\left(x_{2} * x_{1}\right) \in Y_{+}$. Since $Y$ is medial, so $Y_{+}=\{0\}$. Hence, $f\left(x_{1}\right)=f\left(x_{2}\right)$. This proves that $g$ is well defined. Further, it is easy to prove that $g$ is a $\mathrm{BCH}$-homomorphism and makes the following diagram commutative:

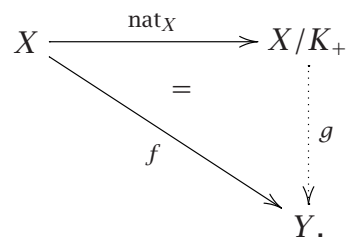

The uniqueness of $g$ follows from the fact that nat ${ }_{X}$ is an onto homomorphism and hence an epimorphism. This completes the proof.

DEFINITION 4.4. Let $X$ and $Y$ be BCH-algebras. A BCH-homomorphism $f$ : $X \rightarrow Y$ is regular if and only if $f(X)=\{f(x): x \in X\}$ is an ideal in $Y$.

Proposition 4.5. Let $f \in \mathrm{BCH}(X, Y)$ be regular. Then for each ideal $I \supseteq$ $\operatorname{Ker} f, f(I)=\{f(x): x \in I\}$ is an ideal of $Y$. Further, for each ideal $K$ of $Y$, $f^{-1}(K)=\{a \mid f(a) \in K, a \in X\}$ is an ideal of $X$.

Proof. Assume $y * b, b \in f(I) \subseteq f(X)$. Then there exists $a \in I$ such that $b=f(a)$. Since $f(X)$ is an ideal, so $y \in f(X)$. Thus, $y=f(x)$ for some $x \in X$. 
Now, $y * b=f(x) * f(a)=f(x * a) \in f(I)$. This implies that $f(x * a)=f(c)$ for some $c \in I$. Thus, we have $f((x * a) * c)=0$ and so $(x * a) * c \in \operatorname{Ker} f \subseteq I$. Since $I$ is an ideal, $x * a \in I$, it follows that $x \in I$. Therefore $y \in f(I)$. Clearly, $0 * y \in f(I)$ for all $y \in f(I)$. Hence, $f(I)$ is an ideal of $Y$.

For the second part, we assume $x * a, a \in f^{-1}(K)$. Then $f(x * a), f(a) \in K$, that is, $f(x) * f(a), f(a) \in K$. Since $K$ is an ideal, it follows that $f(x) \in K$ or $x \in f^{-1}(K)$. Further, note that $0 * a \in f^{-1}(K)$ for all $a \in f^{-1}(K)$. Thus, $f^{-1}(K)$ is an ideal of $X$. This completes the proof.

THEOREM 4.6. Let $f \in \mathrm{BCH}(X, Y)$ be regular. Then there is a one-to-one correspondence between the ideals of $X$ containing Ker $f$ and the ideals of $Y$.

Proof. Let $\Omega=\{I: I \supseteq \operatorname{Ker} f, I$ is an ideal of $X\}$ and $\Omega^{\prime}=\left\{I^{\prime}: I^{\prime}\right.$ is an ideal of $Y$ \}. Let $I \in \Omega$, then by Proposition 4.5, $f(I)$ is an ideal of $Y$. Now, we define $\psi: \Omega \rightarrow \Omega^{\prime}$ as $\psi(I)=f(I)$. Obviously, $\psi$ is well defined. In order to prove the theorem, we show that $\psi$ is one-to-one and onto.

(i) The mapping $\psi$ is one-to-one.

Let $I_{1}, I_{2} \in \Omega$ be such that $\psi\left(I_{1}\right)=\psi\left(I_{2}\right)$. This implies that $f\left(I_{1}\right)=f\left(I_{2}\right)$. Let $x_{1} \in I_{1}$. Then $f\left(x_{1}\right) \in f\left(I_{1}\right)=f\left(I_{2}\right)$. Thus, there exists $x_{2} \in I_{2}$ such that $f\left(x_{1}\right)=f\left(x_{2}\right)$, which gives $x_{1} * x_{2} \in \operatorname{Ker} f \subseteq I_{2}$. Since $x_{2}, x_{1} * x_{2} \in I_{2}$ and $I_{2}$ is an ideal, it follows that $x_{1} \in I_{2}$, proving that $I_{1} \subseteq I_{2}$. Similarly, we can prove that $I_{2} \subseteq I_{1}$. Hence, $I_{1}=I_{2}$. This proves that $\psi$ is one-to-one.

(ii) The mapping $\psi$ is onto.

Let $I^{\prime} \in \Omega^{\prime}$. Then by Proposition 4.5, $f^{-1}\left(I^{\prime}\right)$ is an ideal of $X$. Further, Ker $f \subseteq$ $f^{-1}\left(I^{\prime}\right)$ and $\psi\left(f^{-1}\left(I^{\prime}\right)\right)=I^{\prime}$. Thus, $\psi$ is onto. This completes the proof.

Acknowledgment. M. A. Chaudhry is thankful to the King Fahd University of Petroleum and Minerals, Dhahran, Saudi Arabia, for providing excellent research facilities.

\section{REFERENCES}

[1] M. A. Arbib and E. G. Manes, Arrows, Structures, and Functors, Academic Press, New York, 1975.

[2] M. A. Chaudhry, On BCH-algebras, Math. Japon. 36 (1991), no. 4, 665-676.

[3] M. A. Chaudhry and S. A. Bhatti, On the category of BCI-algebras, Punjab Univ. J. Math. (Lahore) 21 (1988), 105-119.

[4] W. A. Dudek and J. Thomys, On decompositions of BCH-algebras, Math. Japon. 35 (1990), no. 6, 1131-1138.

[5] C. S. Hoo, Quasicommutativity of BCI-algebras, Math. Japon. 32 (1987), no. 5, 741-748.

[6] _ Injectives in the categories of BCK- and BCI-algebras, Math. Japon. 33 (1988), no. 2, 237-246.

[7] C. S. Hoo and P. V. Ramana Murty, Quasicommutative p-semisimple BCI algebras, Math. Japon. 32 (1987), no. 6, 889-894.

[8] Q. P. Hu and X. Li, On BCH-algebras, Math. Sem. Notes Kobe Univ. 11 (1983), no. 2, part 2, 313-320.

[9] _ On proper BCH-algebras, Math. Japon. 30 (1985), no. 4, 659-661. 
[10] Y. Imai and K. Iséki, On axiom systems of propositional calculi. XIV, Proc. Japan Acad. 42 (1966), 19-22.

[11] K. Iséki, An algebra related with a propositional calculus, Proc. Japan Acad. 42 (1966), 26-29.

[12] Some topics from the category of BCK-algebras, Math. Sem. Notes Kobe Univ. 5 (1977), no. 3, 465-468.

[13] On BCI-algebras, Math. Sem. Notes Kobe Univ. 8 (1980), no. 1, 125-130.

[14] K. Iséki and S. Tanaka, Ideal theory of BCK-algebras, Math. Japon. 21 (1976), no. 4, 351-366.

[15] _ An introduction to the theory of BCK-algebras, Math. Japon. 23 (1978), no. 1, 1-26.

[16] K. Iséki and A. B. Thaheem, Note on BCI-algebras, Math. Japon. 29 (1984), no. 2, 255-258.

[17] B. Mitchell, Theory of Categories, Pure and Applied Mathematics, vol. XVII, Academic Press, New York, 1965.

[18] L. Tiande and X. Changchang, p-radical in BCI-algebras, Math. Japon. 30 (1985), no. 4, 511-517.

[19] H. Yutani, Coequalizer in the category of BCK-algebras, Math. Sem. Notes Kobe Univ. 6 (1978), no. 2, 187-188.

[20] Coequalizer in the category of BCK-algebras. II, Math. Sem. Notes Kobe Univ. 7 (1979), no. 1, 143-144.

[21] _ Colimits in the category of BCK-algebras, Math. Japon. 30 (1985), no. 4, 527-534.

Muhammad Anwar Chaudhry: Department of Mathematical Sciences, College of Sciences, King Fahd University of Petroleum and Minerals, Dhahran 31261, Saudi Arabia

E-mail address: chaudhry@kfupm. edu.sa

Hafiz Fakhar-ud-din: Department of Mathematics, Islamia University, Bahawalpur, Pakistan 


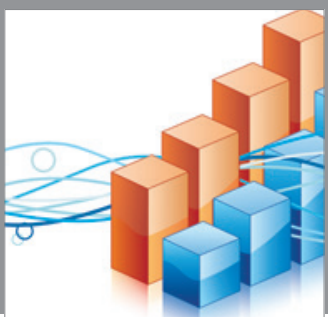

Advances in

Operations Research

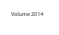

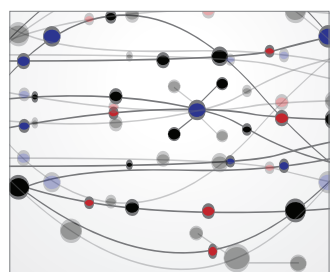

\section{The Scientific} World Journal
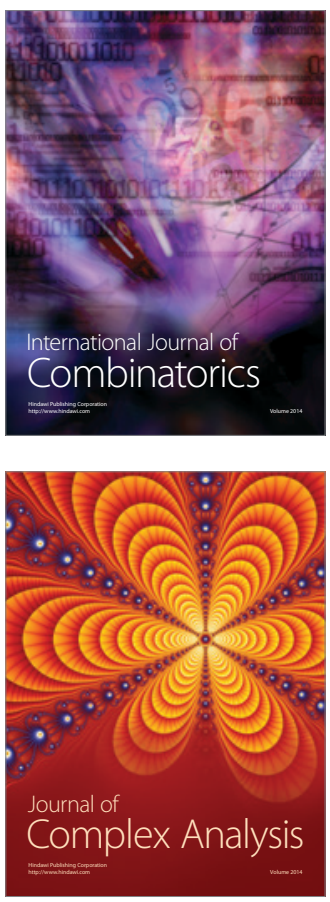

International Journal of

Mathematics and

Mathematical

Sciences
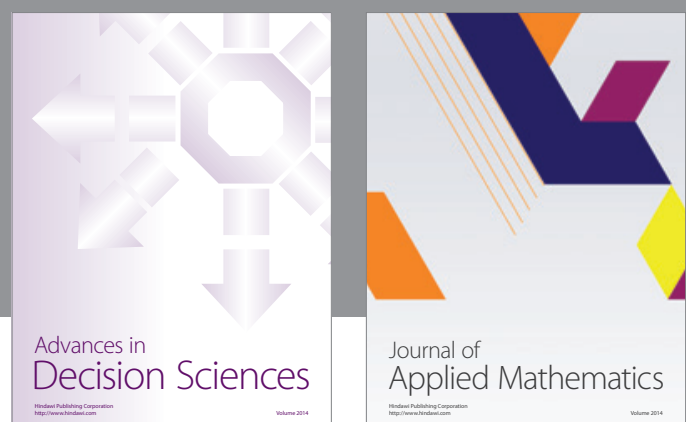

Journal of

Applied Mathematics
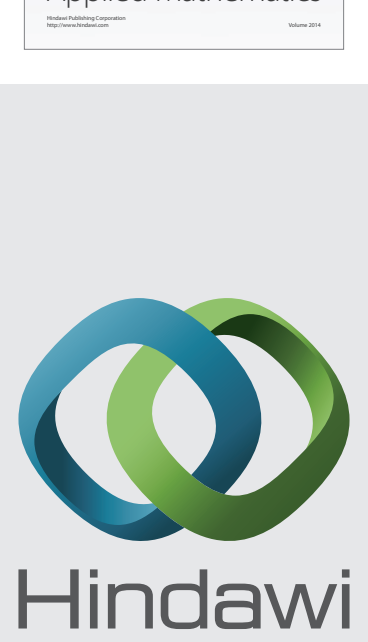

Submit your manuscripts at http://www.hindawi.com
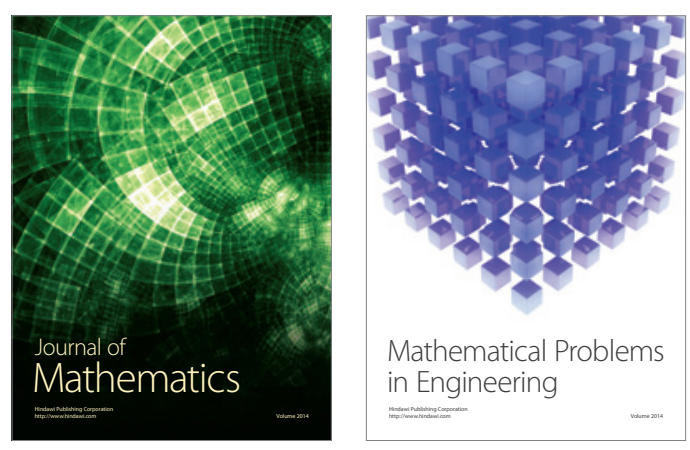

Mathematical Problems in Engineering
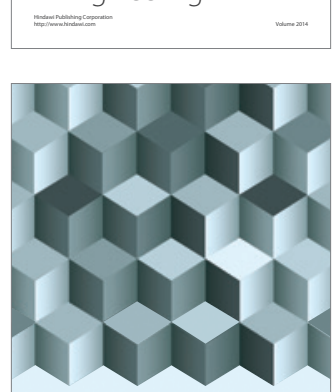

Journal of

Function Spaces
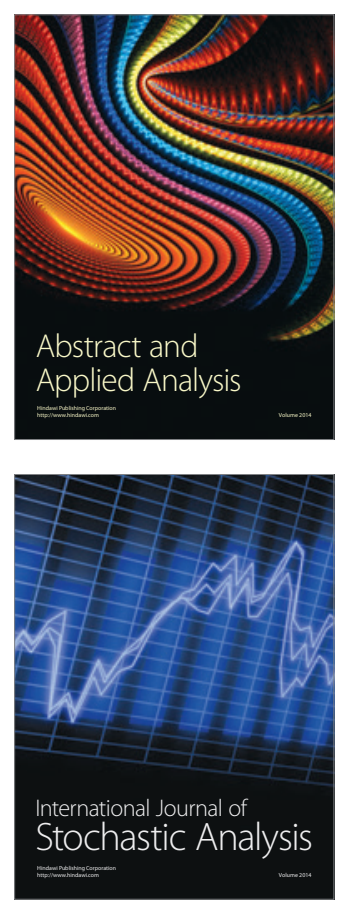

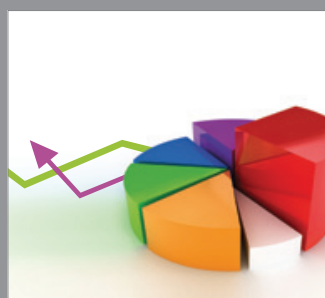

ournal of

Probability and Statistics

Promensencen
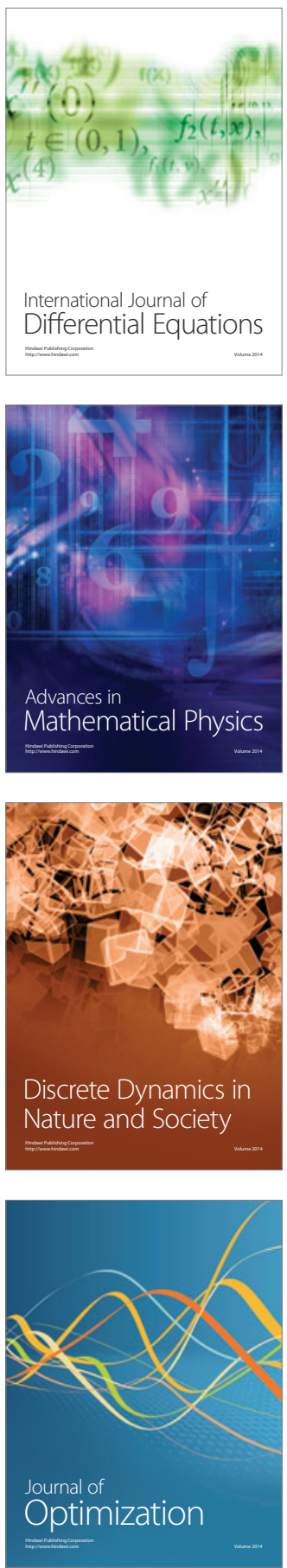